Internat. J. Math. \& Math. Sci

Vol. 23, No. 2 (2000) 131-140

S0161171200000752

(C) Hindawi Publishing Corp.

\title{
A GENERALIZED HANKEL CONVOLUTION ON ZEMANIAN SPACES
}

\section{JORGE J. BETANCOR}

(Received 4 May 1998)

\begin{abstract}
We define a new generalized Hankel convolution on the Zemanian distribution
\end{abstract} spaces of slow growth.

Keywords and phrases. Hankel convolution, Hankel transformation, Zemanian spaces.

2000 Mathematics Subject Classification. Primary 46F12.

1. Introduction. Zemanian (see [17, 19]) investigated the Hankel integral transformation, defined by

$$
h_{\mu}(\phi)(y)=\int_{0}^{\infty}(x y)^{1 / 2} J_{\mu}(x y) \phi(x) d x, \quad y \in(0, \infty),
$$

where $J_{\mu}$ represents the Bessel function of the first kind and of order $\mu$, in spaces of generalized functions. Throughout this paper, $\mu$ is greater than $-1 / 2$.

In [17], it was introduced the space $H_{\mu}$ constituted by all those complex valued and smooth functions $\phi$ on $(0, \infty)$ such that

$$
\gamma_{m, k}^{\mu}(\phi)=\sup _{x \in(0, \infty)}\left|x^{m}\left(\frac{1}{x} D\right)^{k}\left(x^{-\mu-1 / 2} \phi(x)\right)\right|<\infty
$$

for every $m, k \in \mathbb{N} . H_{\mu}$ is endowed with the topology generated by the family $\left\{\gamma_{m, k}^{\mu}\right\}_{m, k \in \mathbb{N}}$ of seminorms and, thus, $H_{\mu}$ is a Fréchet space. The space 0 of multipliers of $H_{\mu}$ was characterized in [3] as follows. A smooth function $f$ on $(0, \infty)$ is in 0 if and only if, for every $k \in \mathbb{N}$, there exists $n \in \mathbb{N}$ such that $\left(1+x^{2}\right)^{n}((1 / x) D)^{k} f(x)$ is a bounded function on $(0, \infty)$ (see [3, Thm. 2.3]). The Hankel transformation $h_{\mu}$ is an automorphism of $H_{\mu}$ (see [19, Thm. 5.4-1]). The dual space of $H_{\mu}$ is denoted by $H_{\mu}^{\prime}$ as usual. The Hankel transformation is defined on $H_{\mu}^{\prime}$ as the transpose of the Hankel transformation on $H_{\mu}$. That is, for each $f \in H_{\mu}^{\prime}$, the Hankel transform $h_{\mu}^{\prime} f$ of $f$ is given by

$$
\left\langle h_{\mu}^{\prime} f, \phi\right\rangle=\left\langle f, h_{\mu} \phi\right\rangle, \quad \phi \in H_{\mu} .
$$

Thus, $h_{\mu}^{\prime}$ is an automorphism of $H_{\mu}^{\prime}$ when it is considered on $H_{\mu}^{\prime}$ the weak* or the strong topology.

Zemanian [18] defined the spaces of functions $B_{\mu, a}, a \in(0, \infty)$ and $B_{\mu}$ as follows. Let $a \in(0, \infty)$. A smooth function $\phi$ on $(0, \infty)$ is in $B_{\mu, a}$ provided that $\phi \in H_{\mu}$ and $\phi(x)=$ $0, x \in(a, \infty)$. This space $B_{\mu, a}$ is equipped with the topology induced by $H_{\mu}$ on it. Thus, $B_{\mu, a}$ is a Fréchet space. Moreover, if $0<a<b$, then $B_{\mu, a}$ is continuously contained in 
$B_{\mu, b}$. The union space $B_{\mu}=\cup_{a>0} B_{\mu, a}$ is endowed with the inductive topology. $B_{\mu}$ is a dense subspace of $H_{\mu}$. The dual space of $B_{\mu}$ is denoted by $B_{\mu}^{\prime}$. In [18, Thm. 1], the Hankel transform $h_{\mu}\left(B_{\mu}\right)$ of $B_{\mu}$ was characterized.

Haimo [12], Hirschman, Jr. [14], and Cholewinski [10] studied the convolution for a variant of the Hankel transformation, which is closely connected to $h_{\mu}$. After straightforward manipulations in the convolution operators defined by the above mentioned authors, a convolution for the transformation $h_{\mu}$ can be obtained. Said convolution operation is defined as follows. Let $f$ and $g$ be measurable functions on $(0, \infty)$. The Hankel convolution $f * g$ of $f$ and $g$ is given by

$$
(f * g)(x)=\int_{0}^{\infty} f(y)\left(\tau_{x} g\right)(y) d y,
$$

where

$$
\left(\tau_{x} g\right)(y)=\int_{0}^{\infty} g(z) D_{\mu}(x, y, z) d z
$$

provided that the above integrals exist, and being

$$
\begin{aligned}
& D_{\mu}(x, y, z) \\
& \quad=\int_{0}^{\infty} t^{-\mu-1 / 2}(x t)^{1 / 2} J_{\mu}(x t)(y t)^{1 / 2} J_{\mu}(y t)(z t)^{1 / 2} J_{\mu}(z t) d t, \quad x, y, z \in(0, \infty) .
\end{aligned}
$$

If $x^{\mu+1 / 2} f$ and $x^{\mu+1 / 2} g$ are in $L_{1}(0, \infty)$, the space of absolutely integrable functions on $(0, \infty)$, then $x^{\mu+1 / 2}(f * g) \in L_{1}(0, \infty)$ and the interchange formula

$$
h_{\mu}(f * g)(x)=x^{-\mu-1 / 2} h_{\mu}(f)(x) h_{\mu}(g)(x), \quad x \in(0, \infty)
$$

holds.

The study of the Hankel convolution on distribution spaces was started by SousaPinto [11]. He defined the Hankel convolution of distributions of compact support on $(0, \infty)$ for $\mu=0$. In the last years, the $*$ convolution was studied in different spaces of generalized functions by Betancor and Marrero (see [4, 5, 6, 7, 15]), Betancor and González [1], and Betancor and Rodríguez-Mesa (see [9, 8]).

The Hankel translation $\tau_{x}$ defines a continuous mapping from $H_{\mu}$ into itself for every $x \in(0, \infty)$ (see [15, Prop. 2.1]). Then the Hankel convolution $T * \phi$ of $T \in H_{\mu}^{\prime}$ and $\phi \in H_{\mu}$ can be defined by

$$
(T * \phi)(x)=\left\langle T, \tau_{x} \phi\right\rangle, \quad x \in(0, \infty) .
$$

In [15, Prop. 3.5], it was proved that $x^{-\mu-1 / 2}(T * \phi) \in \mathcal{O}$ for every $T \in H_{\mu}^{\prime}$ and $\phi \in H_{\mu}$. The subspace $O_{\mu, *}^{\prime}$ of $H_{\mu}^{\prime}$ consisting of the convolution operators in $H_{\mu}$ was characterized in [15, Prop. 4.2] as follows. A functional $T \in H_{\mu}^{\prime}$ belongs to $O_{\mu, *}^{\prime}$ (i.e., $T * \phi \in H_{\mu}$ for every $\phi \in H_{\mu}$ ) if and only if $x^{-\mu-1 / 2} h_{\mu}^{\prime}(T)$ is in 0 . The convolution $S * T$ of $S \in H_{\mu}^{\prime}$ and $T \in \mathcal{O}_{\mu, *}^{\prime}$ is defined in [15].

DEFINITION 1. Let $S \in H_{\mu}^{\prime}$ and $T \in \mathcal{O}_{\mu, *}^{\prime}$. The $*$-convolution $S * T$ of $S$ and $T$ is the element of $H_{\mu}^{\prime}$ defined by

$$
\langle S * T, \phi\rangle=\langle S, T * \phi\rangle, \quad \phi \in H_{\mu} .
$$


If $S \in H_{\mu}^{\prime}$ and $T \in \mathcal{O}_{\mu, *}^{\prime}$, the following extension of the interchange formula (1.7):

$$
h_{\mu}^{\prime}(S * T)(x)=x^{-\mu-1 / 2} h_{\mu}^{\prime}(S) h_{\mu}^{\prime}(T)
$$

holds.

In this paper, inspired in [13], we define the Hankel convolution in a subspace of $H_{\mu}^{\prime} \times H_{\mu}^{\prime}$ that contains $H_{\mu}^{\prime} \times \mathcal{O}_{\mu, *}^{\prime}$. The new convolution generalizes the one defined in [15, Def. 1].

Throughout this paper, $C$ always denotes a suitable positive constant, which is not necessarily the same in each occurrence.

2. The generalized Hankel convolution on $H_{\mu}^{\prime}$. Now, we are going to define a new generalized Hankel convolution on $H_{\mu}^{\prime}$. Let $S$ and $T$ be in $H_{\mu}^{\prime}$. Assume that

(P.1) $(S * \phi)(T * \psi) \in L_{1}(0, \infty)$ for every $\phi, \psi \in H_{\mu}$,

(P.2) $\int_{0}^{\infty} \tau_{x}(T * \phi)(y)(S * \psi)(y) d y=\int_{0}^{\infty}(T * \phi)(y) \tau_{x}(S * \psi)(y) d y$ for every $\phi, \psi \in$ $H_{\mu}$ and $x \in(0, \infty)$.

When $S$ and $T$ satisfy properties (P.1) and (P.2), we say that the pair $(S, T)$ has the $(P)$-property for the sake of simplicity.

Fixing $\psi \in H_{\mu}$, we define the linear mapping $F_{\psi}$ from $H_{\mu}$ into the space $D^{\prime}(0, \infty)$ of the distributions in $(0, \infty)$ by

$$
F_{\psi}(\phi)=(S * \psi)(T * \phi), \quad \phi \in H_{\mu} .
$$

$F_{\psi}$ is a continuous mapping when $D^{\prime}(0, \infty)$ is endowed with the weak* topology. Indeed, according to [15, Prop. 3.5], $x^{-\mu-1 / 2}(S * \phi) \in \mathcal{O}$ and $x^{-\mu-1 / 2}(T * \phi) \in \mathcal{O}$ for each $\phi \in H_{\mu}$. Also, $x^{-\mu-1 / 2}\left(T * \phi_{n}\right) \rightarrow 0$, as $n \rightarrow \infty$, in 0 provided that $\phi_{n} \rightarrow 0$, as $n \rightarrow \infty$, in $H_{\mu}$. Hence, if $\phi_{n} \rightarrow 0$, as $n \rightarrow \infty$, in $H_{\mu}$, then $F_{\psi}\left(\phi_{n}\right) \rightarrow 0$, as $n \rightarrow \infty$, in $D^{\prime}(0, \infty)$. Then we conclude that $F_{\psi}$ is continuous.

Therefore, since $(S, T)$ satisfies (P.1), [16, Thm. 2] implies that $F_{\psi}$ is a continuous mapping from $H_{\mu}$ into $L_{1}(0, \infty)$.

In other words, we have seen that the bilinear mapping

$$
L: H_{\mu} \times H_{\mu} \longrightarrow L_{1}(0, \infty)
$$

defined by

$$
L(\phi, \psi)=(S * \psi)(T * \phi), \quad \psi, \phi \in H_{\mu}
$$

is separately continuous. Then, since $H_{\mu}$ is a Fréchet space, the bilinear form $\mathscr{L}$, defined on $H_{\mu} \times H_{\mu}$ by

$$
\mathscr{L}(\phi, \psi)=\int_{0}^{\infty}(S * \psi)(x)(T * \phi)(x) d x, \quad \psi, \phi \in H_{\mu}
$$

is continuous.

Now, we introduce the linear mapping $\mathbb{R}$ from $H_{\mu}$ into $H_{\mu}^{\prime}$ as follows. For every $\psi \in H_{\mu}, \mathbb{L}(\psi)$ denotes the element of $H_{\mu}^{\prime}$ defined by

$$
\langle\mathbb{L}(\psi), \phi\rangle=\mathscr{L}(\psi, \phi), \quad \phi \in H_{\mu} .
$$


From [5, Lem. 2.2] and by taking into account that $(S, T)$ satisfies (P.2), we have

$$
\mathbb{L}\left(\tau_{y} \psi\right)=\tau_{y}(\mathbb{L} \psi), \quad \psi \in H_{\mu} .
$$

Hence, according to [7, Prop. 1], there exists a unique $R \in H_{\mu}^{\prime}$ such that

$$
\mathbb{L}(\psi)=R * \psi, \quad \psi \in H_{\mu} .
$$

DEFinition 2. Let $S$ and $T \in H_{\mu}^{\prime}$ such that the pair $(S, T)$ satisfies the $(P)$-property. We define the Hankel convolution $S \# T$ of $S$ and $T$ as the unique element of $H_{\mu}^{\prime}$ satisfying

$$
\langle(S \# T) * \psi, \phi\rangle=\int_{0}^{\infty}(S * \psi)(x)(T * \phi)(x) d x, \quad \psi, \phi \in H_{\mu}
$$

Now, we show that Definition 2 applies to a wide class of generalized functions in $H_{\mu}^{\prime}$. Let $m \in \mathbb{Z}$. We consider the space $Y_{m}$ that consists of all those complex valued and smooth functions $f$ on $(0, \infty)$ such that

$$
\sup _{x \in(0, \infty)}\left(1+x^{2}\right)^{m} x^{-\mu-1 / 2}|f(x)|<\infty .
$$

According to [15, proof of Prop. 3.5], if $T \in H_{\mu}^{\prime}$, then there exists $m \in \mathbb{Z}$ for which $T * \phi \in Y_{m}$, for each $\phi \in H_{\mu}$. We say that a functional $T \in H_{\mu}^{\prime}$ is in $\mathbb{Y}_{m}$ when $T * \phi \in Y_{m}$ for every $\phi \in H_{\mu}$.

Proposition 2.1. Let $S \in \mathbb{Y}_{k}$ and $T \in \mathbb{Y}_{m}$. Then $(S, T)$ has the $(P)$-property provided that $m+k<\mu+1$.

Proof. Let $\phi, \psi \in H_{\mu}$. It is easy to see that

$$
(S * \psi)(T * \phi) \in L_{1}(0, \infty) .
$$

Let $x \in(0, \infty)$. We can write $([14,(2)$, p. 308])

$$
\tau_{x}(T * \phi)(y)=\int_{|x-y|}^{x+y} D_{\mu}(x, y, z)(T * \phi)(z) d z, \quad y \in(0, \infty) .
$$

Moreover, since $S \in \mathbb{Y}_{k}$ and $T \in \mathbb{Y}_{m}$, by taking into account [14, (2), p. 310], it follows that

$$
\begin{aligned}
\int_{0}^{\infty} \mid(S & * \psi)(y)\left|\int_{|x-y|}^{x+y} D_{\mu}(x, y, z)\right|(T * \phi)(z) \mid d z d y \\
& \leq C \int_{0}^{\infty} y^{\mu+1 / 2}\left(1+y^{2}\right)^{-k} \int_{|x-y|}^{x+y} D_{\mu}(x, y, z) z^{\mu+1 / 2}\left(1+z^{2}\right)^{-m} d z d y \\
& \leq C x^{\mu+1 / 2} \int_{0}^{\infty}\left(1+y^{2}\right)^{-m-k} y^{2 \mu+1} d y<\infty .
\end{aligned}
$$

Hence, Fubini theorem leads to

$$
\int_{0}^{\infty}(S * \psi)(y) \tau_{x}(T * \phi)(y) d y=\int_{0}^{\infty} \tau_{x}(S * \psi)(y)(T * \phi)(y) d y .
$$

Thus, we conclude that the pair $(S, T)$ has the $(P)$-property. 
In particular, from Proposition 2.1, we can immediately deduce the following.

COROLLARY 2.2. If $S \in H_{\mu}^{\prime}$ and $T \in \mathcal{O}_{\mu, *}^{\prime}$, then $(S, T)$ has the $(P)$-property.

Proof. According to [15, Prop. 4.3], $T * \psi \in H_{\mu}$, for every $\phi \in H_{\mu}$. Hence, $T \in Y_{m}$ for every $m \in \mathbb{Z}$ and, from Proposition 2.1 , we infer that $(S, T)$ has the $(P)$-property.

Now, we establish that the convolution $*$ defined by Definition 1 on $H_{\mu}^{\prime} \times \mathcal{O}_{\mu, *}^{\prime}$ (see [15]) is a special case of the convolution \# given in Definition 2.

Proposition 2.3. Let $S \in H_{\mu}^{\prime}$ and $T \in O_{\mu, *}^{\prime}$. Then $S * T=S \# T$.

Proof. By Corollary 2.2, the pair $(S, T)$ has the $(P)$-property. Moreover, by invoking [15, Props. 3.5 and 4.3], we can write

$$
\begin{aligned}
\langle(S * T) * \psi, \phi\rangle & =\langle S * T, \psi * \phi\rangle=\langle S, T *(\psi * \phi)\rangle \\
& =\langle S,(T * \phi) * \psi\rangle=\langle S * \psi, T * \phi\rangle \\
& =\int_{0}^{\infty}(S * \psi)(x)(T * \phi)(x) d x, \quad \psi, \phi \in H_{\mu} .
\end{aligned}
$$

Thus, we conclude that $S * T=S \# T$.

Next, some algebraic properties of the \#-convolution are proved.

Proposition 2.4. Let $S, T \in H_{\mu}^{\prime}$ and $R \in O_{\mu, *}^{\prime}$. Assume that $(S, T)$ satisfies the (P)-property. Then

(i) $S \# T=T \# S$.

(ii) $(S \# T) \# R=S \#(T \# R)$.

(iii) $T \# \delta_{\mu}=T$, where $\delta_{\mu}$ represents the element of $H_{\mu}^{\prime}$ defined by

$$
\left\langle\delta_{\mu}, \phi\right\rangle=2^{\mu} \Gamma(\mu+1) \lim _{x \rightarrow 0^{+}} x^{-\mu-1 / 2} \phi(x), \quad \phi \in H_{\mu} .
$$

(iv) $S_{\mu}(S \# T)=\left(S_{\mu} S\right) \# T=S \#\left(S_{\mu} T\right)$, where $S_{\mu}$ denotes the Bessel operator $x^{-\mu-1 / 2} D \times$ $x^{2 \mu+1} D x^{-\mu-1 / 2}$.

Proof. (i) It is clear that $(T, S)$ has the $(P)$-property. Moreover, according to [15, Prop. 3.5], for every $\psi, \phi \in H_{\mu}$,

$$
\begin{aligned}
\langle(S \# T) * \psi, \phi\rangle & =\langle S \# T, \psi * \phi\rangle=\langle(S \# T) * \phi, \psi\rangle \\
& =\int_{0}^{\infty}(S * \phi)(x)(T * \psi)(x) d x .
\end{aligned}
$$

Hence, $S \# T=T \# S$.

(ii) By virtue of Proposition 2.3, the pair $(S \# T, R)$ satisfies the $(P)$-property and $(S \# T) \# R=(S \# T) * R$. Moreover, $(S, T * R)$ has the $(P)$-property. Indeed, let $\psi, \phi \in H_{\mu}$. According to [15, Props. 4.3 and 4.7(i)], since $(S, T)$ satisfies the $(P)$-property, we have

$$
(S * \psi)((T * R) * \phi)=(S * \psi)(T *(R * \phi)) \in L_{1}(0, \infty)
$$


and

$$
\begin{aligned}
\int_{0}^{\infty} \tau_{x}((T * R) & * \phi)(y)(S * \psi)(y) d y \\
& =\int_{0}^{\infty} \tau_{x}(T *(R * \phi))(y)(S * \psi)(y) d y \\
& =\int_{0}^{\infty}(T *(R * \phi))(y) \tau_{x}(S * \psi)(y) d y \\
& =\int_{0}^{\infty}((T * R) * \phi)(y) \tau_{x}(S * \psi)(y) d y, \quad x \in(0, \infty) .
\end{aligned}
$$

Also, we can write by [15, Props. 3.5 and 4.7(i)], for each $\phi, \psi \in H_{\mu}$,

$$
\begin{aligned}
\langle((S \# T) * R) * \psi, \phi\rangle & =\langle(S \# T) *(R * \psi), \phi\rangle \\
& =\langle S \# T,(R * \psi) * \phi\rangle \\
& =\langle(S \# T) * \psi, R * \phi\rangle \\
& =\int_{0}^{\infty}(S * \psi)(x)(T *(R * \phi))(x) d x \\
& =\int_{0}^{\infty}(S * \psi)(x)((T * R) * \phi)(x) d x .
\end{aligned}
$$

Thus, we conclude that $(S \# T) * R=S \#(T * R)$.

(iii) It is immediately deduced from [15, Prop. 4.7(iv)] and Proposition 2.3.

(iv) Since $(S, T)$ has the $(P)$-property, $\left(S_{\mu} S, T\right)$ and $\left(S, S_{\mu} T\right)$ also satisfy the same property. Indeed, let $\psi, \phi \in H_{\mu}$. Then, since the Bessel operator $S_{\mu}$ is a continuous operator from $H_{\mu}$ into itself [19, Lem. 5.3-3], by [15, Prop. 4.7(iii)],

$$
\left(\left(S_{\mu} S\right) * \psi\right)(T * \phi)=\left(S *\left(S_{\mu} \psi\right)\right)(T * \phi) \in L_{1}(0, \infty),
$$

and

$$
\begin{aligned}
\int_{0}^{\infty} \tau_{x}(T * \phi) & (y)\left(\left(S_{\mu} S\right) * \psi\right)(y) d y \\
& =\int_{0}^{\infty} \tau_{x}(T * \phi)(y)\left(S *\left(S_{\mu} \psi\right)\right)(y) d y \\
& =\int_{0}^{\infty}(T * \phi)(y) \tau_{x}\left(S *\left(S_{\mu} \psi\right)\right)(y) d y \\
& =\int_{0}^{\infty}(T * \phi)(y) \tau_{x}\left(\left(S_{\mu} S\right) * \psi\right)(y) d y, \quad x \in(0, \infty) .
\end{aligned}
$$

Moreover, by [15, Prop. 2.2(ii)], we get

$$
\begin{aligned}
\left\langle S_{\mu}(S \# T) * \psi, \phi\right\rangle & =\left\langle S_{\mu}(S \# T), \psi * \phi\right\rangle=\left\langle S \# T,\left(S_{\mu} \psi\right) * \phi\right\rangle \\
& =\int_{0}^{\infty}\left(S *\left(S_{\mu} \psi\right)\right)(x)(T * \phi)(x) d x \\
& =\int_{0}^{\infty}\left(\left(S_{\mu} S\right) * \psi\right)(x)(T * \phi)(x) d x, \quad \psi, \phi \in H_{\mu} .
\end{aligned}
$$

Hence, $S_{\mu}(S \# T)=\left(S_{\mu} S\right) \# T$.

To complete the proof of (iv), it is sufficient to take into account (i). 
Our next objective is to prove an interchange formula that relates the Hankel transformation $h_{\mu}^{\prime}$ to the \#-convolution.

First, we need to define the product $T \cdot S$ of $T$ and $S$ belonging to $H_{\mu}^{\prime}$.

As in [4], we say that a sequence $\left\{k_{n}\right\}_{n \in \mathbb{N}} \subset B_{\mu}$ is a Hankel approximated identity when the following three conditions hold for every $n \in N$ :

(i) $k_{n}(x) \geq 0, x \in(0, \infty)$;

(ii) $k_{n}(x)=0, x \notin((1 / n+1),(1 / n))$;

(iii) $\int_{0}^{1 / n} k_{n}(x) x^{\mu+1 / 2} d x=2^{\mu} \Gamma(\mu+1)$.

Three useful properties of the Hankel approximated identities follow.

Proposition 2.5 ([2, Prop. 1] and [6, proof of Prop. 2.4, p. 1148]). Let $\left\{k_{n}\right\}_{n \in \mathbb{N}}$ be a Hankel approximated identity. Then, we have

(i) For every $a>0, y^{-\mu-1 / 2} h_{\mu}\left(k_{n}\right)(y) \rightarrow 1$, as $n \rightarrow \infty$, uniformly in $(0, a)$, and there exists $M>0$ such that $\left|y^{-\mu-1 / 2} h_{\mu}\left(k_{n}\right)(y)\right| \leq M, n \in \mathbb{N}$ and $y \in(0, \infty)$.

(ii) For every $\phi \in H_{\mu}, k_{n} * \phi \rightarrow \phi$, as $n \rightarrow \infty$, in $H_{\mu}$.

(iii) For every $T \in H_{\mu}^{\prime}, T * k_{n} \rightarrow T$, as $n \rightarrow \infty$, in the strong topology of $H_{\mu}^{\prime}$.

Let $T$ and $S$ be in $H_{\mu}^{\prime}$. We say that $R \in B_{\mu}^{\prime}$ is the product $x^{-\mu-1 / 2} T \cdot S$ and we write $R=$ $x^{-\mu-1 / 2} T \cdot S$ if for every Hankel approximated identity $\left\{k_{n}\right\}_{n \in \mathbb{N}}, x^{-\mu-1 / 2}\left(T * k_{n}\right) S \rightarrow R$ and $x^{-\mu-1 / 2}\left(S * k_{n}\right) T \rightarrow R$, as $n \rightarrow \infty$, in the weak* topology of $B_{\mu}^{\prime}$.

Note that if $T, S \in H_{\mu}^{\prime}$ and there exists the product $x^{-\mu-1 / 2} T \cdot S$ of $T$ and $S$, then also there exists the product $x^{-\mu-1 / 2} S \cdot T$ of $S$ and $T$, and $x^{-\mu-1 / 2} T \cdot S=x^{-\mu-1 / 2} S \cdot T$. Moreover, if $T \in H_{\mu}$ and $S \in H_{\mu}^{\prime}$, then

$$
\left\langle x^{-\mu-1 / 2} T \cdot S, \phi\right\rangle=\left\langle S, x^{-\mu-1 / 2} T \phi\right\rangle, \quad \phi \in B_{\mu} .
$$

Indeed, let $\left\{k_{n}\right\}_{n \in \mathbb{N}}$ be a Hankel approximated identity. Then we have, by Proposition 2.5(ii) and (iii),

$$
\begin{aligned}
& \left\langle x^{-\mu-1 / 2}\left(T * k_{n}\right) S, \phi\right\rangle=\left\langle S, x^{-\mu-1 / 2}\left(T * k_{n}\right) \phi\right\rangle \longrightarrow\left\langle S, x^{-\mu-1 / 2} T \phi\right\rangle, \quad \text { as } n \rightarrow \infty, \\
& \left\langle x^{-\mu-1 / 2}\left(S * k_{n}\right) T, \phi\right\rangle=\left\langle S * k_{n}, x^{-\mu-1 / 2} T \phi\right\rangle \longrightarrow\left\langle S, x^{-\mu-1 / 2} T \phi\right\rangle, \quad \text { as } n \longrightarrow \infty,
\end{aligned}
$$

for every $\phi \in B_{\mu}$.

Hence, the product that we have defined between two elements of $H_{\mu}^{\prime}$ extends the usual product of a function in $H_{\mu}$ by a distribution in $H_{\mu}^{\prime}$.

Proposition 2.6 (The interchange formula). Let $S, T \in H_{\mu}^{\prime}$. Assume that the pair $(S, T)$ has the $(P)$-property. Then, we have

$$
h_{\mu}^{\prime}(S \# T)=x^{-\mu-1 / 2} h_{\mu}^{\prime}(S) \cdot h_{\mu}^{\prime}(T) .
$$

Proof. We only have to prove that, for every $\phi \in B_{\mu}$,

$$
\left\langle x^{-\mu-1 / 2}\left(h_{\mu}^{\prime}(S) * k_{n}\right) h_{\mu}^{\prime}(T), \phi\right\rangle \rightarrow\left\langle h_{\mu}^{\prime}(S \# T), \phi\right\rangle, \quad \text { as } n \rightarrow \infty,
$$

where $\left\{k_{n}\right\}_{n \in \mathbb{N}}$ is a Hankel approximated identity.

Let $\phi \in B_{\mu}$ and let $\left\{k_{n}\right\}_{n \in \mathbb{N}}$ be a Hankel approximated identity. There exists $a>0$ such that $\phi \in B_{\mu, a}$. Choose $b>a$ and $\chi \in B_{\mu}$ such that $\chi(x)=x^{\mu+1 / 2}, x \in(0, b)$. 
According to [19, Thm. 5.4-1], $h_{\mu}(\phi) \in H_{\mu}$ and $h_{\mu}(\chi) \in H_{\mu}$. Hence, since $(S, T)$ has the $(P)$-property, from Proposition 2.5(i), it follows that

$$
\begin{aligned}
& \int_{0}^{\infty}\left(S * h_{\mu}(\phi)\right)(x)\left(T * h_{\mu}(x)\right)(x) d x \\
& \quad=\lim _{n \rightarrow \infty} \int_{0}^{\infty}\left(S * h_{\mu}(\phi)\right)(x)\left(T * h_{\mu}(x)\right)(x) x^{-\mu-1 / 2} h_{\mu}\left(k_{n}\right)(x) d x .
\end{aligned}
$$

Suppose that $\left\{\alpha_{n}\right\}_{n \in \mathbb{N}}$ is also a Hankel approximated identity. By [15, Prop. 4.5], we can write

$$
\begin{aligned}
\left\langle x^{-\mu-1 / 2}\right. & \left.h_{\mu}\left(\alpha_{m}\right)\left(S * h_{\mu}(\phi)\right),\left(T * h_{\mu}(\chi)\right) x^{-\mu-1 / 2} h_{\mu}\left(k_{n}\right)\right\rangle \\
& =\left\langle h_{\mu}^{\prime}\left(x^{-\mu-1 / 2} h_{\mu}\left(\alpha_{m}\right)\left(S * h_{\mu}(\phi)\right)\right), h_{\mu}\left(\left(T * h_{\mu}(\chi)\right) x^{-\mu-1 / 2} h_{\mu}\left(k_{n}\right)\right)\right\rangle \\
& =\left\langle\left(x^{-\mu-1 / 2} \phi h_{\mu}^{\prime}(S)\right) * \alpha_{m},\left(x^{-\mu-1 / 2} \chi h_{\mu}^{\prime}(T)\right) * k_{n}\right\rangle, \quad n, m \in \mathbb{N} .
\end{aligned}
$$

Since $\left(T * h_{\mu}(\chi)\right) x^{-\mu-1 / 2} h_{\mu}\left(k_{n}\right) \in H_{\mu}$ ([15, Prop. 3.5]), $n \in \mathbb{N}$, also $\left(x^{-\mu-1 / 2} \chi h_{\mu}^{\prime}(T)\right) *$ $k_{n} \in H_{\mu}, n \in \mathbb{N}$. Hence, by Proposition 2.5(iii), we have, for each $n \in \mathbb{N}$,

$$
\begin{aligned}
\left\langle\left(x^{-\mu-1 / 2} \phi h_{\mu}^{\prime}(S)\right)\right. & \left.* \alpha_{m},\left(x^{-\mu-1 / 2} \chi h_{\mu}^{\prime}(T)\right) * k_{n}\right\rangle \\
& \longrightarrow\left\langle x^{-\mu-1 / 2} \phi h_{\mu}^{\prime}(S),\left(x^{-\mu-1 / 2} \chi h_{\mu}^{\prime}(T)\right) * k_{n}\right\rangle, \quad \text { as } m \longrightarrow \infty
\end{aligned}
$$

Moreover, since $(S, T)$ has the $(P)$-property and according to Proposition 2.5(i), one has, for every $n \in \mathbb{N}$,

$$
\begin{aligned}
\left\langle x^{-\mu-1 / 2}\right. & \left.h_{\mu}\left(\alpha_{m}\right)\left(S * h_{\mu}(\phi)\right),\left(T * h_{\mu}(\chi)\right) x^{-\mu-1 / 2} h_{\mu}\left(k_{n}\right)\right\rangle \\
& =\int_{0}^{\infty} x^{-\mu-1 / 2} h_{\mu}\left(\alpha_{m}\right)(x)\left(S * h_{\mu}(\phi)\right)(x)\left(T * h_{\mu}(\chi)\right)(x) x^{-\mu-1 / 2} h_{\mu}\left(k_{n}\right)(x) d x \\
& \longrightarrow \int_{0}^{\infty}\left(S * h_{\mu}(\phi)\right)(x)\left(T * h_{\mu}(\chi)\right)(x) x^{-\mu-1 / 2} h_{\mu}\left(k_{n}\right)(x) d x \\
& =\left\langle S * h_{\mu}(\phi),\left(T * h_{\mu}(\chi)\right) x^{-\mu-1 / 2} h_{\mu}\left(k_{n}\right)\right\rangle, \quad \text { as } m \rightarrow \infty .
\end{aligned}
$$

Hence, for every $n \in \mathbb{N}$,

$$
\begin{aligned}
\left\langle S * h_{\mu}(\phi),\right. & \left.\left(T * h_{\mu}(\chi)\right) x^{-\mu-1 / 2} h_{\mu}\left(k_{n}\right)\right\rangle \\
& =\left\langle x^{-\mu-1 / 2} \phi h_{\mu}^{\prime}(S),\left(x^{-\mu-1 / 2} \chi h_{\mu}^{\prime}(T)\right) * k_{n}\right\rangle .
\end{aligned}
$$

On the other hand, since $\chi(x)=x^{\mu+1 / 2}, x \in(0, b)$, being $b>a$, there exists $n_{0} \in \mathbb{N}$ such that

$$
\begin{aligned}
& \left\langle x^{-\mu-1 / 2} \phi h_{\mu}^{\prime}(S),\left(x^{-\mu-1 / 2} \chi h_{\mu}^{\prime}(T)\right) * k_{n}\right\rangle \\
& \quad=\left\langle h_{\mu}^{\prime}(S), x^{-\mu-1 / 2} \phi\left(h_{\mu}^{\prime}(T) * k_{n}\right)\right\rangle \quad \text { for every } n \geq n_{0} .
\end{aligned}
$$

Moreover, according to [15, Prop. 3.5],

$$
\begin{aligned}
\left\langle h_{\mu}^{\prime}(S \# T), \phi\right\rangle & =\left\langle S \# T, h_{\mu}(\phi)\right\rangle=\left\langle S \# T, h_{\mu}\left(x^{-\mu-1 / 2} \chi \phi\right)\right\rangle \\
& =\left\langle S \# T, h_{\mu}(\phi) * h_{\mu}(\chi)\right\rangle=\left\langle(S \# T) * h_{\mu}(\phi), h_{\mu}(\chi)\right\rangle .
\end{aligned}
$$


By combining (2.26), (2.30), and (2.32), it follows that

$$
\begin{aligned}
\left\langle h_{\mu}^{\prime}(S \# T), \phi\right\rangle & =\left\langle(S \# T) * h_{\mu}(\phi), h_{\mu}(x)\right\rangle \\
& =\lim _{n \rightarrow \infty} \int_{0}^{\infty}\left(S * h_{\mu}(\phi)\right)(x)\left(T * h_{\mu}(\chi)\right)(x) x^{-\mu-1 / 2} h_{\mu}\left(k_{n}\right)(x) d x \\
& =\lim _{n \rightarrow \infty}\left\langle x^{-\mu-1 / 2} h_{\mu}^{\prime}(S)\left(h_{\mu}^{\prime}(T) * k_{n}\right), \phi\right\rangle .
\end{aligned}
$$

Thus, the proof is complete.

REMARK. Propositions 2.4 and 2.6 are extensions of [15, Props. 4.5 and 4.7].

ACKNOWLEDGEMENT. This work is partially supported by DGICYT grant PB 940591 (Spain).

\section{REFERENCES}

[1] J. J. Betancor and B. J. Gonzàlez, A convolution operation for a distributional Hankel transformation, Studia Math. 117 (1995), no. 1, 57-72. MR 97c:46049. Zbl 837.46029.

[2] J. J. Betancor, M. Linares, and J. M. R. Méndez, The Hankel transform of integrable Boehmians, Applicable Anal. 58 (1995), no. 3-4, 367-382. MR 97h:44010. Zbl 831.44004.

[3] J. J. Betancor and I. Marrero, Multipliers of Hankel transformable generalized functions, Comment. Math. Univ. Carolin. 33 (1992), no. 3, 389-401. MR 94f:46051. Zbl 801.46047.

[4] _ The Hankel convolution and the Zemanian spaces $\beta_{\mu}$ and $\beta_{\mu}^{\prime}$, Math. Nachr. 160 (1993), 277-298. MR 95j:46042. Zbl 796.46023.

[5] _ Some properties of Hankel convolution operators, Canad. Math. Bull. 36 (1993), no. 4, 398-406. MR 95f:46064. Zbl 795.46024.

[6] Structure and convergence in certain spaces of distributions and the generalized Hankel convolution, Math. Japon. 38 (1993), no. 6, 1141-1155. MR 95j:46043. Zbl 795.46023.

[7]_, Algebraic characterization of convolution and multiplication operators on Hankeltransformable function and distribution spaces, Rocky Mountain J. Math. 25 (1995), no. 4, 1189-1204. MR 97a:46054. Zbl 853.46034.

[8] J. J. Betancor and L. Rodríguez-Mesa, Hankel convolution on distribution spaces with exponential growth, Studia Math. 121 (1996), no. 1, 35-52. MR 98e:46047. Zbl 862.46021.

[9] _ On Hankel convolution equations in distribution spaces, Rocky Mountain J. Math. 29 (1999), no. 1, 93-114. Zbl 990.48075.

[10] F. M. Cholewinski, A Hankel convolution complex inversion theory, Mem. Amer. Math. Soc. 58 (1965), 67. MR 31\#5043. Zbl 137.30901.

[11] J. de Sousa-Pinto, A generalised Hankel convolution, SIAM J. Math. Anal. 16 (1985), no. 6, 1335-1346. MR 87g:44004. Zbl 592.46038.

[12] D. T. Haimo, Integral equations associated with Hankel convolutions, Trans. Amer. Math. Soc. 116 (1965), 330-375. MR 32\#2847. Zbl 135.33502.

[13] Y. Hirata and H. Ogata, On the exchange formula for distributions, J. Sci. Hiroshima Univ. Ser. A 22 (1958), 147-152. MR 22\#897. Zbl 088.08603.

[14] I. I. Hirschman, Jr., Variation diminishing Hankel transforms, J. Analyse Math. 8 (1960/1961), 307-336. MR 28\#433. Zbl 099.31301.

[15] I. Marrero and J. J. Betancor, Hankel convolution of generalized functions, Rend. Mat. Appl. (7) 15 (1995), no. 3, 351-380. MR 96m:46072. Zbl 833.46026.

[16] K. Yoshinaga and H. Ogata, On convolutions, J. Sci. Hiroshima Univ. Ser. A 22 (1958), 15-24. MR 21\#6532. Zbl 088.33202.

[17] A. H. Zemanian, A distributional Hankel transformation, SIAM J. Appl. Math. 14 (1966), 561-576. MR 34\#1807. Zbl 154.13803. 
[18] _ The Hankel transformation of certain distributions of rapid growth, SIAM J. Appl. Math. 14 (1966), 678-690. MR 35\#2093. Zbl 154.13804.

[19]__ Generalized Integral Transformations, Pure and Applied Mathematics, vol. XVIII, Interscience Publishers [John Wiley \& Sons, Inc.], New York, London, Sydney, 1968. MR 5410991.

BeTANCOR: DePaRTAMENTO DE ANÁLISIS MATEMÁtico, UNiVERSIDAD DE LA LAGUNA, 38271LA LAGUNA. TENERIFE, ISLAS CANARIAS, ESPAÑA

E-mail address: jbetanco@u11.es 


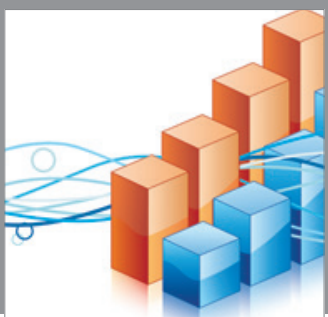

Advances in

Operations Research

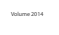

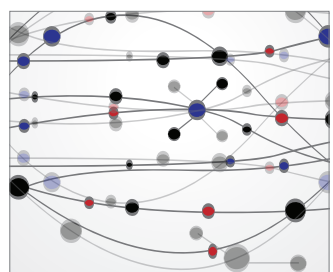

\section{The Scientific} World Journal
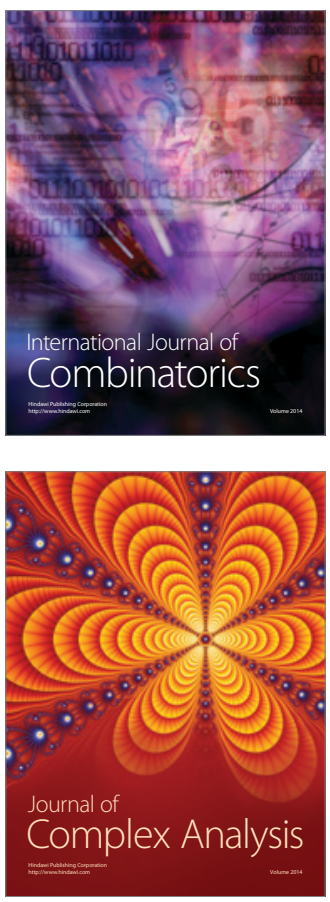

International Journal of

Mathematics and

Mathematical

Sciences
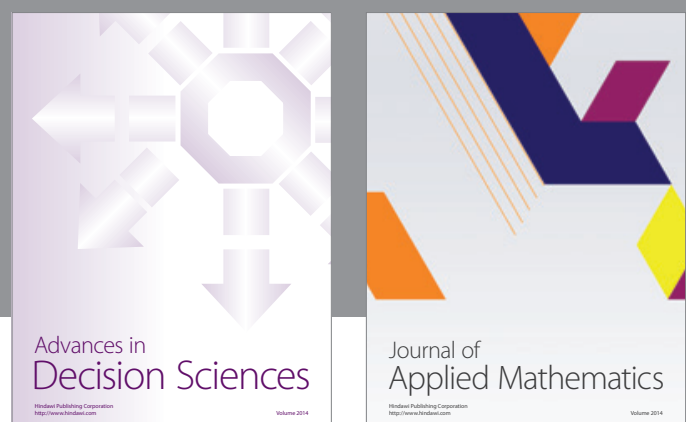

Journal of

Applied Mathematics
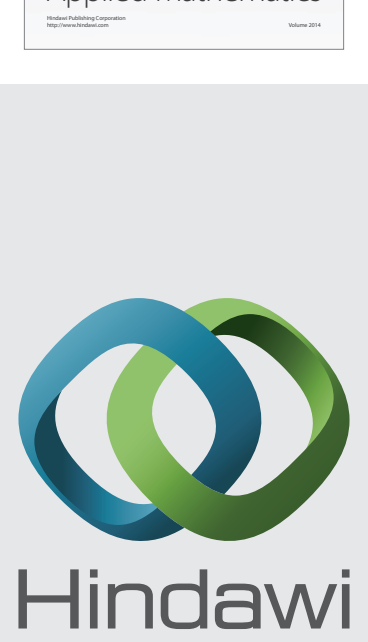

Submit your manuscripts at http://www.hindawi.com
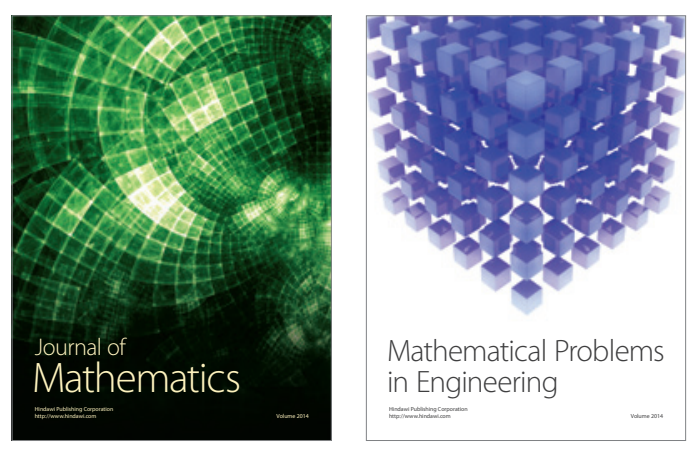

Mathematical Problems in Engineering
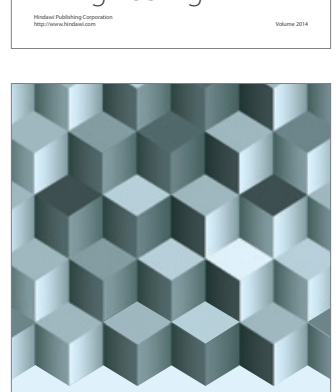

Journal of

Function Spaces
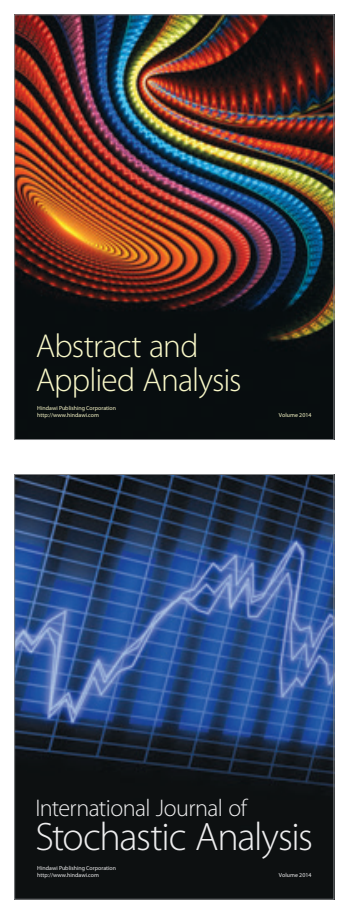

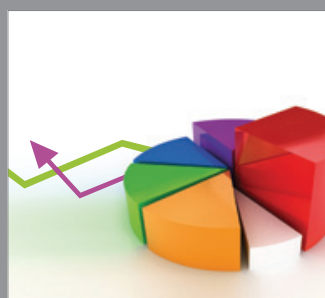

ournal of

Probability and Statistics

Promensencen
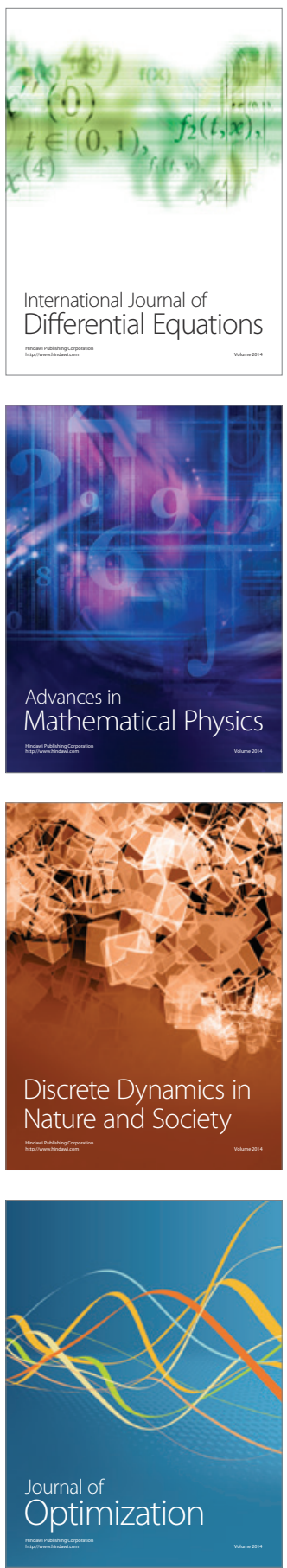